\title{
Computational Modeling in Pyrometallurgy: Part I
}

\author{
Q.G. REYNOLDS ${ }^{1,3}$ and M.A. RHAMDHANI (1) ${ }^{2,4}$ \\ 1.-MINTEK Pyrometallurgy Division, University of Stellenbosch, Randburg, South Africa. \\ 2.-FPD (Fluid and Process Dynamics) Research Group, Swinburne University of \\ Technology, Melbourne, Australia \\ 3.—e-mail: QuinnR@mintek.co.za. 4.—e-mail: ARhamdhani@swin.edu.au
}

\section{INTRODUCTION}

Pyrometallurgy is the subtle art and science of using high temperatures to process raw materials into valuable products. This is performed in several key stages, including pre-treatment, chemical reactions, separation of product and waste streams, and product refining. The interior of pyrometallurgical vessels in all stages is an extreme and hazardous environment, with process temperatures well in excess of $1000^{\circ} \mathrm{C}$. As a result, it is a uniquely challenging subject for experimental study and measurement, and in many cases the task of improving our fundamental understanding and engineering knowledge of pyrometallurgical processes falls solely to mathematical, numerical, and computational models of the various phenomena at work.

When developing such models, the level of complexity present in the governing physics and chemistry is, however, a formidable obstacle. It is not uncommon for a single pyrometallurgical unit operation to exhibit behavior related to simultaneous heat transfer and thermal radiation, multiphase and free surface fluid flow, phase thermodynamics and thermochemistry, solid-liquid-gas phase changes, flow and motion of granular media, electromagnetic stirring and heating effects, transonic and supersonic flow, thermo-mechanical cycling and material fracture, and many others. Strong coupling between these different phenomena generally mean that they cannot be studied in isolation and must be solved as part of integrated multiphysics models. Additionally relevant behavior may occur over an extremely broad range of length and time scales, from micrometers to tens of meters and from milliseconds to days.*

(Received June 29, 2021; accepted June 30, 2021; published online July 14, 2021)

*Quinn Reynolds and M Akbar Rhamdhani are Guest Editors for the Pyrometallurgy Committee of TMS and coordinated the topic Computational Modeling in Pyrometallurgy in this issue.
Such complexity has historically made it very difficult indeed to develop efficient numerical algorithms for the study of pyrometallurgy using computational modeling methods. However, sustained increases in available computer power combined with new advances in both computational hardware and software are for the first time starting to give us a glimmer of insight into this most intractable of engineering challenges. In this special topic, we present nine papers that highlight those computational modeling advances.

\section{PAPERS IN THIS TOPIC}

Computational modeling of dispersed-phase systems is a not straightforward problem. In general, some approximation methods or "closures" must be applied to the dispersed phase in order to reduce the computational complexity of the overall model to manageable levels. Assessing the impact of different choices of closures on the results obtained for a given problem is an important and often neglected part of due diligence in modeling. In "Effect of Drag Laws and Turbulence Models on CFD Modeling of the Bubble Behavior and Fluid Flow in RH Reactor" by $\mathrm{Q}$. Cao et al., the authors demonstrate this by exploring the effect of different fluid turbulence and drag force closures on computational fluid dynamics (CFD) models of a reactor unit used in steelmaking.

The level of process complexity in pyrometallurgy can often seem daunting. Although it is tempting (and instructive to a degree) to isolate and study individual parts of the physics and chemistry on their own, important coupling and interaction effects between various phenomena may easily be missed in this way. In "An Overall Furnace Model for the Silicomanganese Process," M. Sparta et al. have demonstrated the utility of a computational multiphysics model for silicomanganese smelting which combines granular flow, fluid flow, heat transfer, and thermochemical reaction behavior into a single integrated framework. 
The development of electric arc furnaces for pyrometallurgy dates back to the nineteenth century and they remain an established technology today. Plasma arcs are effective sources of thermal and mechanical stirring, and permit the prosecution of many smelting processes which would otherwise be impossible. Arcs are, however, tremendously complex phenomena to model, originating as they do from tight coupling between electromagnetic, fluid flow, and heat transfer effects. In "Momentum Transfer from Arc to Slag Bath in a DC Ilmenite Smelting Furnace-A Computational Analysis," T. Makgoale et al. present a comprehensive analysis of a promising method for reducing the computational complexity of arcs in integrated multiphysics models.

Continuous casting is one of the most highintensity processes to be found in pyrometallurgy. Molten steel is poured into a cooled metal mold open at the bottom, and solidifies into a solid ingot as it is continuously drawn out. Continuous casters require precision operation and control to achieve the high throughputs required in the steel industry, and catastrophic failure of the unit can result if the mold shell is fractured during operation. Computational multiphysics models are valuable as virtual prototypes and in silico experiments for fine-tuning the design of pyrometallurgical equipment, as demonstrated in "Effect of Final Electromagnetic Stirring on Flow, Solidification, and Solute Transport in Continuous-Casting Bloom” by Q. Fang et al.

One of the most powerful ways in which computational models can be used in pyrometallurgy is as a complement to laboratory and industrial experiments, in order to provide deeper fundamental understanding and interpretation of results which often require enormous effort to generate. In "Quantitative Evaluation of Slag Corrosion on MgO-C Refractory by Experiment and Numerical Simulation" by Q. Wang et al., the authors have developed a computational model of a common refractory corrosion test and compared experimental and numerical results to both validate their model and improve the understanding of the processes involved in $\mathrm{MgO}-\mathrm{C}$ refractory corrosion.

Aluminium production via molten salt electrolysis in the Hall-Héroult process is one of the most established pyrometallurgical industries. It requires highly specialised furnace vessels and electrode designs in order to optimize the flow of large electric currents and the separation of the metallic product. Computational tools are of great use in analyzing and evaluating these designs, as is demonstrated by $\mathrm{T}$. Li et al. in "Effects of Height Difference of the Recycled Stub on the Anode Performance in Hall-Héroult Cell.”

The production of copper in pyrometallurgical units such as smelters and converters is an interesting process in that it generally treats two raw materials in different phases (the copper ore as a liquid melt, and the oxygen as a pure gas or mixture). These phases must be brought into intimate contact to ensure the occurrence of the desired thermochemical reactions. The method and location of injection of the gas into the melt pool varies considerably between different furnace designs; in "Computational Fluid Dynamics Study on Enhanced Circulation Flow in a Side-blown Copper Smelting Furnace," Y. Xiao et al. make use of multiphase computational fluid dynamics models to assess the impact of changes to the injection nozzles in a side-injected copper smelter.

Movement of reacting particles is very complex in a highly dynamic basic oxygen furnace during steelmaking. In "Numerical Investigation of Lime Particle Motion in Steelmaking BOF Process," Y. Xiao and coauthors attempted to evaluate lime particle motion in the top and bottom blowing BOF reactor using $3 \mathrm{D}$ numerical modeling. The results provide some insight on how the particles initially move to the side before coming back to the center and gradually dispersing in the slag.

Many complex phenomena and processes are occurring during aluminium electrolysis in a cell. In "Mode Coupling Analysis of Interfacial Stability and Critical Anode-Cathode Distance in a 500-kA Aluminum Electrolysis Cell, M. Li et al. attempted to quantify and evaluate the magneto-hydrodynamic interfacial instability. This work puts forward a number of frequencies that promote instability in the cell. It is also demonstrated that increasing the anode-cathode distance promotes the stability of the cell.

All titles and authors of the articles published under the topic "Computational Modeling in Pyrometallurgy" in the September 2021 issue (vol. 73, no. 9) of JOM can be accessed via the journal's page at: http:// link.springer.com/journal/11837/73/9/page/1.

Publisher's Note Springer Nature remains neutral with regard to jurisdictional claims in published maps and institutional affiliations. 